\title{
Brief Communication: Contrast-stretching- and histogram-smoothness-based synthetic aperture radar image enhancement for flood map generation
}

\author{
F. Nazir ${ }^{1}$, M. M. Riaz ${ }^{2}$, A. Ghafoor ${ }^{1}$, and F. Arif ${ }^{1}$ \\ ${ }^{1}$ Military College of Signals, National University of Sciences and Technology (NUST), Islamabad, Pakistan \\ ${ }^{2}$ Centre for Advanced Studies in Telecommunication (CAST), COMSATS, Islamabad, Pakistan
}

Correspondence to: A. Ghafoor (abdulghafoor-mcs@nust.edu.pk)

Received: 22 September 2013 - Published in Nat. Hazards Earth Syst. Sci. Discuss.: 6 August 2014

Revised: 4 January 2015 - Accepted: 9 January 2015 - Published: 5 February 2015

\begin{abstract}
Synthetic-aperture-radar-image-based flood map generation is usually a challenging task (due to degraded contrast). A three-step approach (based on adaptive histogram clipping, histogram remapping and smoothing) is proposed for generation of a more visualized flood map image. The pre- and post-flood images are adaptively histogram equalized. The hidden details in difference image are enhanced using contrast-based enhancement and histogram smoothing. A fast-ready flood map is then generated using equalized pre-, post- and difference images. Results (evaluated using different data sets) show significance of the proposed technique.
\end{abstract}

\section{Introduction}

Flood detection/mapping is desirable in variety of applications like disaster management, risk/damage assessment and rehabilitation process. Flood mapping/monitoring techniques use pre- and post-synthetic aperture radar (SAR) images to classify non flooded and flooded (inundated) areas (Kussel et al., 2011; Nazir et al., 2013, 2014). However, SAR images often contain speckle noise, which results in unwanted artifacts and contrast degradation. Different pre-processing techniques (like spatial- and transform-based filtering) are applied (before flood detection) to overcome these issues.

Visual interpretation (Chambenoit et al., 2003) requires the user's involvement for the identification of flooded areas (which is not always feasible). Semi-automatic segmentation-based flood detection techniques generally re- quire empirical seed point selection (Dellepiane et al., 2010; Martinis et al., 2011). Thresholding-based unsupervised flood classification (Moser and Serpico, 2006) does not work under complex environmental conditions (Pulvirenti et al., 2011). The texture-matching-based scheme (Zhao et al., 2011) suffers from high computational time due to overlapping features.

Schumann et al. (2009) have applied different processing steps to generate inundation maps require reliable calibration and verification. Complex coherence maps are used for the analysis of SAR data for flood monitoring, however, optical images are required for result verification (Chini et al., 2012). The Dellepiane and Angiati (2012) flood monitoring technique sometimes highlights unnecessary details in the flood map. Matgen et al. (2011) combines region growing and radiometric thresholding for extraction of the flood map. Giustarini et al. (2013) proposed an automated flood extent extraction scheme using backscatter thresholding, region growing and change detection techniques.

A three-step approach (based on adaptive histogram clipping (AHC), histogram remapping (HR) and histogram smoothness (HS)) is proposed for generation of a more visualized RGB flood map image. The proposed technique follows the basic methodology proposed by Dellepiane and Angiati (2012) with certain modifications/improvements. More specifically the proposed scheme first enhances the preand post-flood images using adaptive histogram equalization (AHE). The difference image (generated from pre- and postflood images) is passed through a contrast-enhancement (CE) and constraint-dependent HS to enhance the hidden details. 


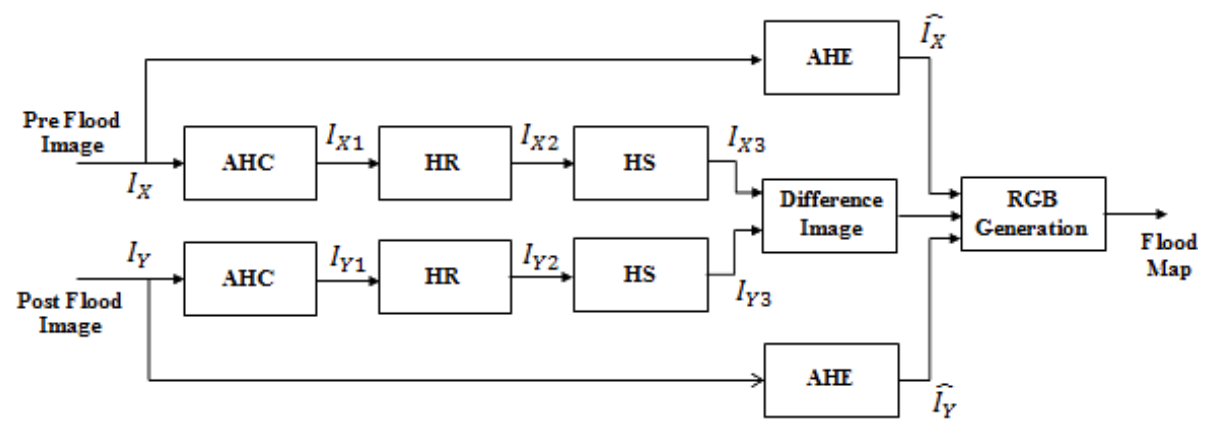

Figure 1. Flow chart of proposed algorithm.

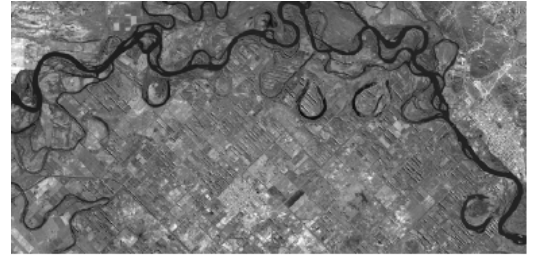

(a)

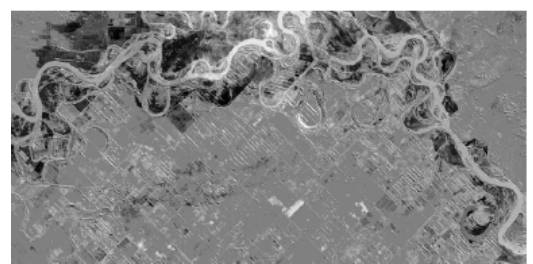

(d)

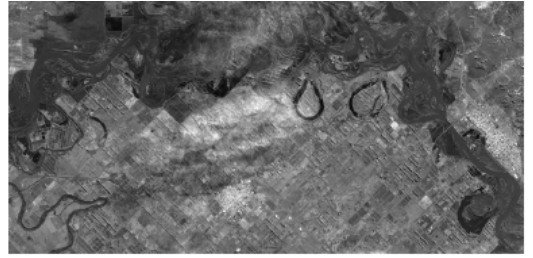

(b)

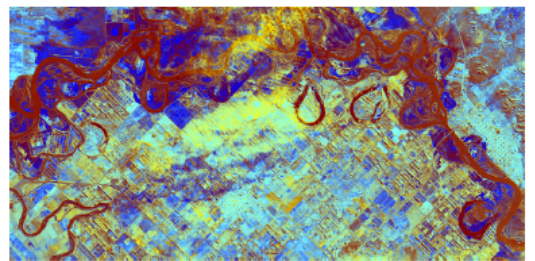

(e)

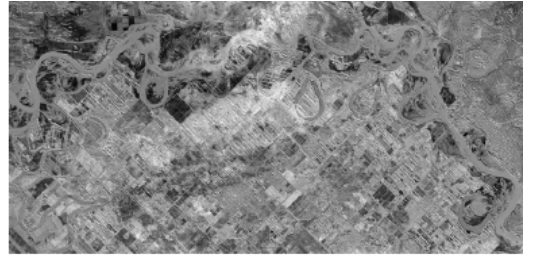

(c)

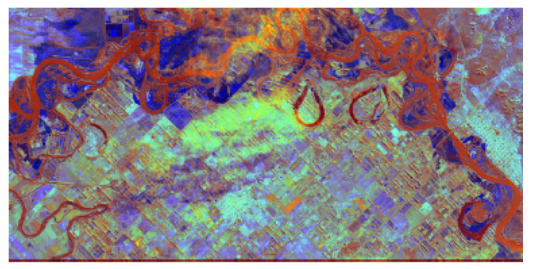

(f)

Figure 2. Original images of Choele Choel, Argentina observed by "Daichi" (ALOS: Advanced Land Observing Satellite): (a) pre-flood image acquired on 29 April 2006. (b) Post-flood image acquired on 30 July 2006. (c) Difference image obtained using the Dellepiane and Angiati (2012) technique. (d) Difference image obtained using proposed technique. (e) Fast-ready map generated using the Dellepiane and Angiati (2012) technique. (f) Fast-ready map generated using proposed technique.

A RGB flood map is then generated using processed pre-, post- and difference images. Simulation results on different data sets are evaluated using visual and quantitative analysis. The proposed technique not only provides better visualization (of flooded areas), but also it has better quantitative results as compared to Dellepiane and Angiati (2012) technique.

\section{Proposed methodology}

Let $\mathbf{I}_{X(l, m)}$ be the pre-image, $\mathbf{I}_{Y(l, m)}$ be the post-image and $\mathbf{I}_{Z(l, m)}$ be the difference image, where $l \in[0, \ldots, L-1]$ and $m \in[0, \ldots, M-1]$. Figure 1 shows the block diagram of proposed technique.

The histogram of pre-image $\mathbf{I}_{X}$ is clipped (identical to Dellepiane and Angiati, 2012, but with a low percentile value i.e., $q=0.40$ ) to obtain $\mathbf{I}_{X 1}$. Note that low percentile values can remove desired details, whereas high percentile values can highlight unwanted details. Therefore, an optimal value of percentile value is used to preserve the intensity values (which contribute to flooding).

The HR is then applied on $\mathbf{I}_{X 1}$ to rescale the intensity range between 0 and 255 using simple linear scaling (Dellepiane and Angiati, 2012) to obtain $\mathbf{I}_{X 2}$.

In next step, HS is applied to improve the visualization by preserving the details. In contrast to the Dellepiane and Angiati (2012) technique, which uses simple histogram equalization (HE), we use HS to maintain the natural look, to suppress unwanted artifacts and to enhance the desired details.

In HS, a smoothness constraint is added to remove abrupt changes using backward difference $K$ (Arici and Dikbas, 2009). The principle is to minimize the difference between modified $\boldsymbol{h}_{X 2_{m}}$ and current $\boldsymbol{h}_{2}$ histograms such that the modified histogram is also closer to the uniform histogram $\boldsymbol{h}_{X 2_{u}}$ with an additional penalty term $\beta\left\|K h_{2}\right\|$ (added for smoothness) i.e.,

$\min \left\|\boldsymbol{h}_{X 2_{m}}-\boldsymbol{h}_{2}\right\|_{2}^{2}+\alpha\left\|\boldsymbol{h}_{X 2_{m}}-\boldsymbol{h}_{X 2_{u}}\right\|_{2}^{2}+\beta\left\|K h_{2}\right\|_{2}^{2}$. 
Table 1. Quantitative comparison.

\begin{tabular}{llcccc}
\hline Data set & Technique & $\chi^{\mathrm{M}}$ & $\chi^{\mathrm{V}}$ & $\chi^{\mathrm{G}}$ & $\chi^{\mathrm{S}}$ \\
\hline \multirow{2}{*}{ Argentina } & Dellepiane and Angiati (2012) & 0.1411 & 0.0551 & 1.1773 & 0.5268 \\
& Proposed & 0.2482 & 0.0346 & 0.9465 & 0.4337 \\
\hline \multirow{2}{*}{ Japan } & Dellepiane and Angiati (2012) & 0.2743 & 0.0560 & 0.9198 & 0.5259 \\
& Proposed & 0.5472 & 0.0382 & 0.7334 & 0.4234 \\
\hline
\end{tabular}

The solution to above constraint problem is (Arici and Dikbas, 2009),

$\boldsymbol{h}_{X 2_{m}}=\left((1+\alpha) \mathbf{I}+\beta(K)^{T} K\right)^{-1} \times\left(\boldsymbol{h}_{2}+\alpha \boldsymbol{h}_{X 2_{u}}\right)$,

where, $\mathbf{I}$ is identity matrix, $\alpha, \beta$ are the contrast enhancement and smoothness parameters (chosen empirically as 0.5 and 1000, respectively). The histogram $\boldsymbol{h}_{X 2_{m}}$ is then used as a mapping function for $\mathrm{HE}$ to generate image $\mathbf{I}_{X 3}$. All three steps (AHC, HR and HS) are applied on $\mathbf{I}_{Y}$ (post-flood image) to generate $\mathbf{I}_{Y 3}$.

The difference image $\mathbf{I}_{Z}$ is generated as,

$\mathbf{I}_{Z}(l, m)=128+\frac{\mathbf{I}_{X 3}(l, m)-\mathbf{I}_{Y 3}(l, m)}{2}$.

The pre- and post-flood images $\mathbf{I}_{X}$ and $\mathbf{I}_{Y}$ are passed through CE step to produce $\hat{\mathbf{I}}_{X}$ and $\hat{\mathbf{I}}_{Y}$, respectively. The reason for skipping the first two steps is to preserve intensity values of pre- and post-images. The processed pre- and postflood images for difference image generation is to remove the intensities that contribute very minimally in flooded areas. Finally $\mathbf{I}_{Z}, \hat{\mathbf{I}}_{X}$ and $\hat{\mathbf{I}}_{Y}$ are combined (by assigning red, blue and green bands) to generate a fast-ready map. The level of red color is high for pixels whose pre-value dominates and vice versa. Red (medium to dark) color represents the permanent water (like rivers) while the dark blue color represents flooded areas.

\section{Simulation and results}

Existing and proposed techniques are evaluated on different SAR images. Figure $2 \mathrm{a}$ and $\mathrm{b}$ shows pre- and post-flood images of Choele Choel, Argentina observed by "Daichi" (ALOS) on 29 April and 30 July 2006 respectively. Figure $2 \mathrm{c}$ and $\mathrm{d}$ show the difference images obtained using both the Dellepiane and Angiati (2012) and the proposed techniques, respectively. In Fig. 2c, the ground details are more prominent, while Fig. 2d comparatively highlights the major required details. The differences in detail contribute significantly to their respective RGB image (shown in Fig. 2e and f). In Fig. 2e, a very high contribution of irrelevant details (of difference image) is visible (for instance, the blue color at the center and at the top right corner). Figure $2 \mathrm{f}$ provides better visibility of flooded areas around river (at the top center) and

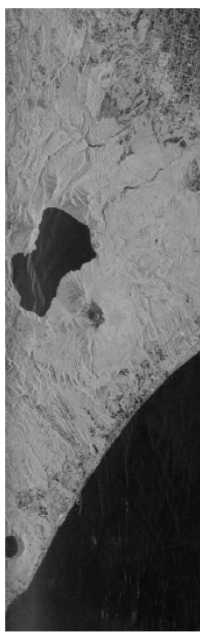

(a)

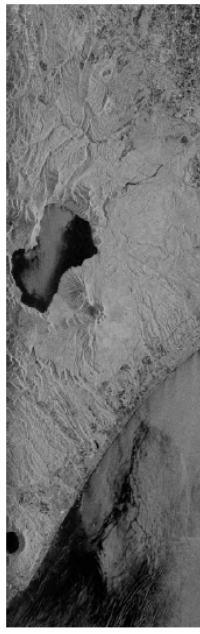

(b)

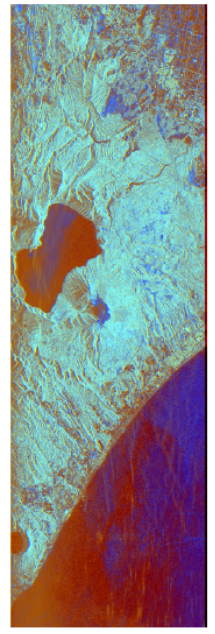

(c)

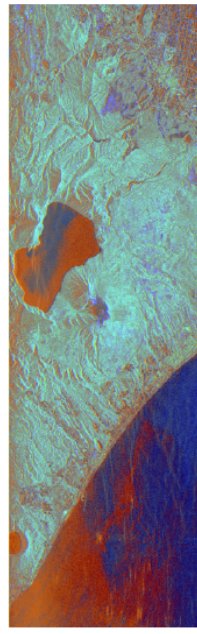

(d)
Figure 3. Evaluation of results using images of Tomakomai, Japan. (a) Pre-image acquired on 19 August 2006. (b) Post-image acquired on 19 August 2006. (c) Fast-ready map generated using the Dellepiane and Angiati (2012) technique. (d) Fast-ready map generated using proposed technique.

low flooded areas (at the center (below river) and top right corner).

Figure $3 \mathrm{a}$ and $\mathrm{b}$ show the images of Tomakomai, Japan, acquired by Phased Array Type L-band SAR (PALSAR) using $\mathrm{H} / \mathrm{V}$ and V/V polarization on 19 August 2006 respectively. Figure $3 \mathrm{c}$ is the RGB flood map generated using the Dellepiane and Angiati (2012) technique. The flood map (in Fig. 3c) highlights some irrelevant details which contribute to flooding (blue colored areas at the right center of the image). Figure $3 \mathrm{~d}$ shows the flood map generated using the proposed technique. Figure $3 d$ preserves the natural effect of image as compared to Fig. 3c.

For quantitative analysis of existing and proposed schemes, different metrics like mutual information $\chi^{\mathrm{M}}$, image variance $\chi^{\mathrm{V}}$, color gradients $\chi^{\mathrm{G}}$ and image saturation $\chi^{\mathrm{S}}$ are used. Since the difference image aims to contain complementary information present in both the (pre- and post-flood) images, the high value of $\chi^{\mathrm{M}}$ is desirable. On the other hand, it is desirable that the generated flood map is smooth and does not contain sharp color transitions (since the flood regions are smooth in nature); therefore, low values of $\chi^{\mathrm{V}}, \chi^{\mathrm{G}}$ 
are required. The flood map must not contain color saturation since it degrades the visual quality of images, so $\chi^{\mathrm{S}}$ should be low. Table 1 shows the quantitative comparison of existing and proposed techniques on both the data sets. It can be observed that the proposed technique provides better quantitative results as compared to the Dellepiane and Angiati (2012) technique.

\section{Conclusions}

A technique for flood map generation based on contrast stretching and histogram smoothing is presented. Different processing steps based on contrast stretching and histogram smoothness are applied on pre-, post- and difference images to generate flood maps. Simulation results show improved visualization by maintaining the natural smoothness. The quantitative comparison also verify the superiority of proposed scheme.

Acknowledgements. We are thankful to the editor and anonymous reviewers for their useful suggestions in improving the quality of this paper.

Edited by: H. Kreibich

Reviewed by: three anonymous referees

\section{References}

Arici, T. and Dikbas, S.: A histogram modification framework and its application for image contrast enhancement, IEEE T. Image Process., 18, 1921-1935, 2009.

Chambenoit, N., Classeau, E. T., and Rudant, J. P.: Performance assessment of multitemporal SAR images' visual interpretation, IEEE International Geoscience and Remote Sensing Symposium, Toulouse, France, 21-25 July 2003, 6, 3911-3913, doi:10.1109/IGARSS.2003.1295311, 2003.

Chini, M., Pulvirenti, L., and Pierdicca, N.: Analysis and interpretation of the Cosmo-SkyMed observations of the 2011 Japan Tsunami, IEEE Geosci. Remote S., 9, 467-471, 2012.

Dellepiane, S. and Angiati, E.: A new method for crossnormalization and multitemporal visualization of SAR images for the detection of flooded areas, IEEE T. Geosci. Remote, 50, 2765-2779, 2012.
Dellepiane, S., Angiati, E., and Vernazza, G.: Processing and segmentation of cosmo-skymed images for flood monitoring, International Geoscience and Remote Sensing Symposium, Honolulu, USA, 25-30 July 2010, 4807-4810, 2010.

Giustarini, L., Hostache, R., Schumann, G. J.-P., Matgen, P., Bates, P. D., and Mason, D. C.: A change detection approach to flood mapping in urban areas using TerraSAR-X, IEEE T. Geosci. Remote, 51, 2417-2430, 2013.

Kussul, N., Shelestov, A., and Skakun, S.: Use of Satellite and InSitu Data to Improve Sustainability, NATO Science for Peace and Security Series C: Environmental Security, 9-29, 2011.

Martinis, S., Twele, A., and Voigt, S.: Unsupervised extraction of flood-induced backscatter changes in SAR data using Markov image modeling on irregular graphs, IEEE T. Geosci. Remote, 49, 251-263, 2011.

Matgen, P., Hostache, R., Schumann, G., Pfister, L., Hoffmann, L., and Savenije, H. H. G.: Towards an automated SAR-based flood monitoring system: Lessons learned from two case studies, Phys. Chem. Earth, 36, 241-252, 2011.

Moser, G. and Serpico, S. B.: Generalized minimum-error thresholding for unsupervised change detection from SAR amplitude imagery, IEEE T. Geosci. Remote, 44, 2972-2982, 2006.

Nazir, F., Riaz, M. M., Ghafoor, A., and Arif, F.: Contrast enhancement based flood monitoring, IEEE International Symposium on Intelligent Signal Processing and Communication Systems, Okinawa, Japan, 12-15 November, 2013.

Nazir, F., Riaz, M. M., Ghafoor, A., and Arif, F.: Flood detection/monitoring using adjustable histogram equalization technique, ScientificWorldJournal, 1-7, 2014.

Pulvirenti, L., Pierdicca, N., Chini, M., and Guerriero, L.: An algorithm for operational flood mapping from Synthetic Aperture Radar (SAR) data using fuzzy logic, Nat. Hazards Earth Syst. Sci., 11, 529-540, doi:10.5194/nhess-11-529-2011, 2011.

Schumann, G., Baldassarre, G. D., and Bates, P.: The utility of spaceborne radar to render flood inundation maps based on multialgorithm ensembles, IEEE T. Geosci. Remote, 47, 2801-2807, 2009.

Zhao, M., Shang, H., Huang, W., Zou, L., and Zhang, Y.: Flood area extraction from rgb aerophotograph based on chromatic and textural analysis, International Conference on Advanced Geographic Information Systems, Appliations and Services GeoProcessing, 46-52, 2011. 